\title{
Performance Analysis on Flow Through S Shaped Diffusers
}

\author{
G. K. Agrawal ${ }^{1}$, Mrs. U. S. Powle ${ }^{2}$ \\ ${ }^{1}$ Department of Mechanical Engineering, Government Engineering College, Bilaspur, Chhattisgarh, India-495009 \\ ${ }^{2}$ Department of Mechanical Engineering, Indian Institute Technology Bombay, Maharastra, India-400076 \\ e-mail: gka1966@yahoo.com \\ Received Nov'30, 2019; received in revised form Jan'23, 2020; Accepted Jan’23, 2020
}

\begin{abstract}
Many times, due to space limitations it is necessary to decelerate and turn fluid simultaneously. For such requirement curved diffusers are being used. Five $S$ shaped diffusers of turning angle $120^{\circ}$ were fabricated. The area ratio was $2.5,3.5,4.5,5.5$ and 6.5 . The pressure on the outer wall is more than that on the inner wall in the first curvature and less in the second curvature. Variation in actual pressure recovery coefficient $(\mathrm{Cp})$ and pressure recovery efficiency $(\eta)$ is not very much within the range of Reynolds number tested for a particular diffuser. As area ratio increases value of $\mathrm{Cp}$ and $\eta$ also increases. The optimum area ratio for $120^{\circ}$ diffuser is 4.5 .
\end{abstract}

Key words- Pressure recovery coefficient; Pressure recovery efficiency; Diffuser; Reynolds number

Broad Area- Mechanical Engineering

Sub Area- Fluid Mechanics

\section{Introduction}

In internal fluid flow systems, it is often required to convert the kinetic energy of the flow to the potential energy in the form of pressure rise. Diffusers are extensively used for this purpose in various engineering systems. The diffuser which is commonly used is straight or curved. The straight diffusers are commonly used in turbines, pumps, fans, automotive and aircraft handling system, axial flow and centrifugal compressor, winds and water tunnels etc. Sometimes due to space limitations it is necessary to decelerate and turn fluid simultaneously. For such requirement curved diffusers are being used. Along with many other applications, this need occurs in vanned diffusers of centrifugal compressor stages, in steam turbine exhaust hoods, in interconnect ducting between components in gas turbine, in ducting downstream of axial compressors, inlet gates of dams and turbine flumes etc. $\mathrm{S}$ shaped diffusers find its most common application in air intake system of air craft engines, apart from space limitation requirements. Galle and Binder, [1] studied flow through a diffuser with and without an exit length. Kline, [2] investigated the flow regimes in straight diffusers. Kline et al., [3] defined the four common optimum problems in diffuser design. Performance data and flow characteristics for subsonic straight plane walled diffusers was presented by Waitman et al., [4]. Carlson et al., [5] studied the effect of wall shape on flow regimes and performance in straight diffusers. Senoo and Nishi, [6] investigated the flow separation in straight walled diffusers by a boundary layer calculation. Experimental evaluation of free stream turbulence on the performance of a subsonic straight diffuser was made by Hoffman, [7]. Thakur et al., [8] used flow visualization technique to determine line of appreciable stall for straight square diffusers at a constant Reynolds number over wide range of diffuser geometries. Ward Smith et al., [9] experimentally investigated the use of screens and perforated plates to control the velocity 
profile emerging from wide at diffuser. Systematic map of flow regime in curved diffusing passage was presented by Fox and Cline, [10]. Sagi and Johnston, [11] presented research paper about the design and performance of curved diffusers. Thakur et al., [12] presented a systematic map of flow regime in curved square diffuser using wall tuft technique of flow visualization. Experiments to determine the flow structure in a large area $(\mathrm{AR}=3.4)$ curved diffuser $\left(\Delta \beta=90^{\circ}\right.$ with elliptical centre line was carried out by Majumadar et al., [13]. Majumadar et al., [14] made detailed flow velocity, pressure and turbulence intensity measurements in a $90^{\circ}$ turn curved diffuser of inlet aspect ratio 6 and area ratio 2. Weng and Guo, [15] presented a new approach of swirl control in an S shaped diffuser, called the 'Automatic Adjustable Blade (AAB) method. Tabhane, [16] investigated the flow on $S$ shaped diffuser with turning angle $30^{\circ}$ and $90^{\circ}$. Varshney, [17] studied flow on $60^{\circ}$ turning angle $\mathrm{S}$ shaped diffuser.

\section{Basic Theory}

Basic theory is discussed under the following heads (i) Geometrical Parameters, (ii) Performance Parameters

(i) Geometrical Parameters:

The performance of a diffuser is largely dependent upon its geometrical parameters. These are area ratios, divergence angle and non dimensional length (Fig.1). The performance of two different diffusers can be compared by keeping some of their geometrical parameters same. The equation for the effective semi divergence angle $\left(\varphi_{\text {eff }}\right.$ and area ratio (AR) for the $S$ shaped diffuser can be given as

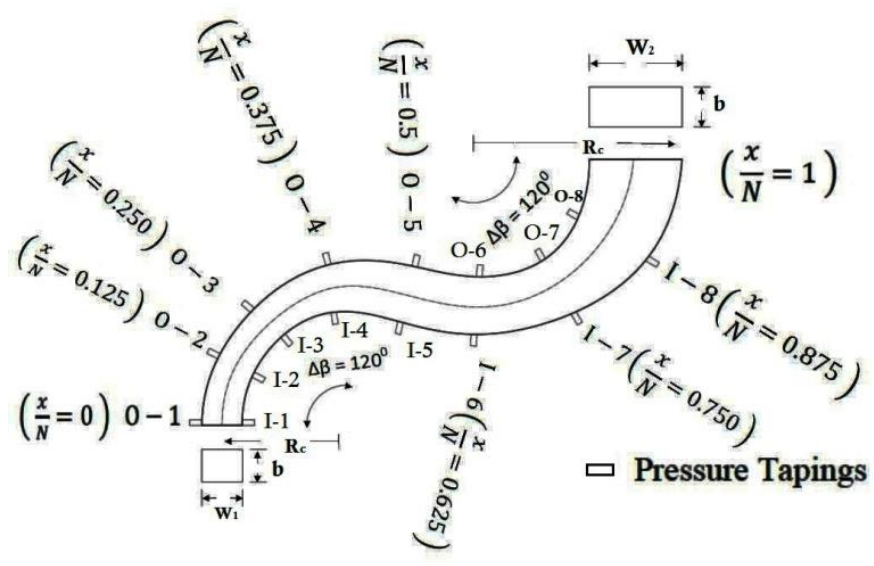

Figure 1. Schematic of S- shaped diffuser with turning angle $120^{\circ}$

$\varphi_{\mathrm{eff}}=\tan ^{-1}\left(\mathrm{~W}_{2}-\mathrm{W}_{1}\right) / 2 \mathrm{~N}$

$\mathrm{W}_{2}=\mathrm{W}_{1}+2 \mathrm{~N} \tan \varphi_{\mathrm{eff}}$

(2)

Where $\mathrm{W}_{1}$ and $\mathrm{W}_{2}$ are the height of the diffuser at inlet and outlet respectively and $\mathrm{N}$ is the axial length.

Area Ratio (AR)

$\mathrm{A}_{2} / \mathrm{A}_{1}=\mathrm{W}_{2} / \mathrm{W}_{1}=1+2 \mathrm{Ntan} \varphi_{\mathrm{eff}} / \mathrm{W}_{1}$

Aspect Ratio AS=b/ $\mathrm{W}_{1}$

(4)

Where $b$ is the distance between parallel diverging walls

The axial length $N$, radius of curvature $R_{c}$ and turning angle $\Delta \beta$ are related by the following equation for a circular arc centre line axis.

$$
\mathrm{R}_{\mathrm{c}}=(360 / \Delta \beta) \times(\mathrm{N} / 2 / 2 \pi)
$$

\section{(ii) Performance parameters:}

The basic function of a diffuser is to convert the dynamic pressure into pressure energy. Relative 
measures of pressure recovery capability are obtained by comparing the measured pressure rise with ideal pressure rise that can be achieved in a particular diffuser geometry. The ideal pressure rise is never achieved in practice because of various energy losses. The losses are due to friction and expansion. The frictional losses in a diffuser are dependent upon length and size of passage, fluid velocity, surface roughness etc. The inlet condition like distorted inlet velocity profiles and the divergence angle contribute to expansion losses. The common performance parameters used to express the diffuser performance are:

(a) Actual pressure recovery coefficient: It is denoted by $\mathrm{C}_{\mathrm{p}}$ and is defined as the ratio of the actual static pressure rise in the diffuser to the average kinetic energy at the inlet.

$$
\mathrm{C}_{\mathrm{p}}=\left(\mathrm{p}_{2}-\mathrm{p}_{1}\right) / 0.5 \rho \mathrm{V}_{1}^{2}
$$

(b)Ideal pressure recovery coefficient: It is denoted by $\mathrm{C}_{\mathrm{pi}}$ and is defined as the ratio of the ideal static pressure rise in the diffuser to the average kinetic energy at the inlet of the diffuser.

$$
\begin{gathered}
\mathrm{C}_{\mathrm{pi}}=0.5 \rho \mathrm{V}_{1}^{2}\left(1-\left(\mathrm{A}_{2} / \mathrm{A}_{1}\right)^{2}\right) / 0.5 \rho \mathrm{V}_{1}{ }^{2} \\
(7) \\
\mathrm{C}_{\mathrm{pi}}=1-1 /(\mathrm{AR})^{2}
\end{gathered}
$$

(c) Pressure recovery efficiency: It is also called pressure recovery efficiency and is denoted by $\eta$. It is defined as the ratio of static pressure rise of the real fluid to that of an ideal fluid from the same geometry at the same flow rate.

$$
\eta=C_{p} / C_{p i}=\left(p_{2}-p_{1}\right) / 0.5 \rho V_{1}^{2}\left(1-\left(A_{2} / A_{1}\right)^{2}\right)
$$

(d)Reynolds number: It is an important dimensionless number which determines nature of flow (laminar or turbulent). It is the ratio of inertia force to viscous force and is given by

$$
\mathrm{Re}=\rho \mathrm{VD} / \mu=\mathrm{VD} / \nu
$$

Where $\rho=$ density of fluid

$\mathrm{V}=$ average velocity of fluid, $\mathrm{D}=$ hydraulic diameter $=4$ cross section area/ wetted perimeter;

$\mu=$ dynamic viscosity and $v=$ kinematic viscosity

\section{Experimental Programme}

Five S shaped diffusers of turning angle $120^{\circ}$ of plastic acrylic sheet were fabricated. The area ratio was $2.5,3.5,4.5,5.5$ and 6.5. The inlet area was $40 \mathrm{X} 40 \mathrm{~mm}^{2}$. The radius of curvature was $191 \mathrm{~mm}$ and length along centre line was 800 $\mathrm{mm}$. The experimental set up as shown in fig. 2 comprised of a blower, a settling chamber, a flow control valve, a long square duct and finally the diffuser test section. The pressure at different sections of duct and diffuser was measured with the help of $U$ tube manometers. The temperature of working fluid at the inlet and exit of diffuser was measured by means of two thermocouples. The flow of working fluid through square duct was measured with the help of pitot tube. 


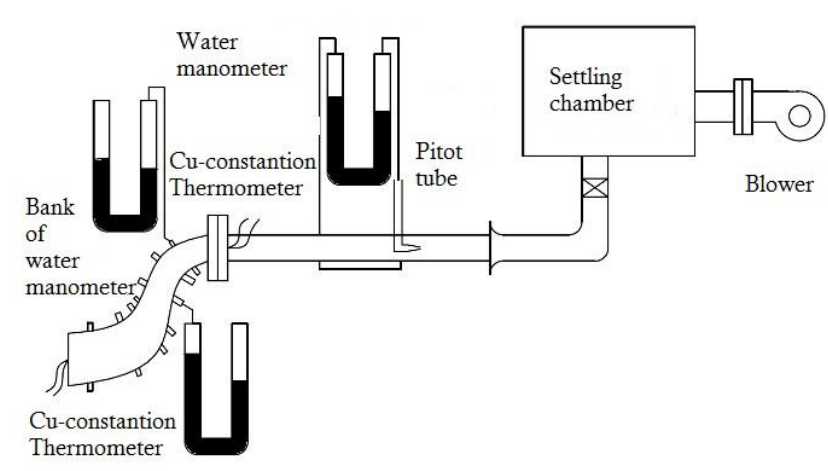

Figure 2. Experimental Setup

The performance tests were conducted on all the diffusers with air as working medium. Temperature is of great importance in any pneumatic experiment. All the tests were carried out at steady state temperature. The steady state temperature was obtained by running the blower for about 15 minutes. Then the pressure, velocity and temperature measurements were undertaken by varying blower discharge, hence the inlet velocity and Reynolds number. The experiments were carried out with diffusers discharging to atmosphere over a wide range of Reynolds number

\section{Results and Discussion}

Pressure distribution on the outer and inner walls: Pressure distribution curve is shown in Fig. 3. For $\mathrm{AR}=2.5$ and $\Delta \beta=120^{\circ}$ pressure on outer wall first increases and reaches above atmospheric till $37.5 \%$ of non dimensional length, then drops down till $62.5 \%$ of non dimensional length and then again increases up to atmospheric pressure. This may be due to flow separation at $37.5 \%$ of non dimensional length and reattachment at $62.5 \%$ of non dimensional length. On the inner wall pressure continuously increases and reaches above atmospheric up to $87.5 \%$ of non dimensional and again reduced to atmospheric.

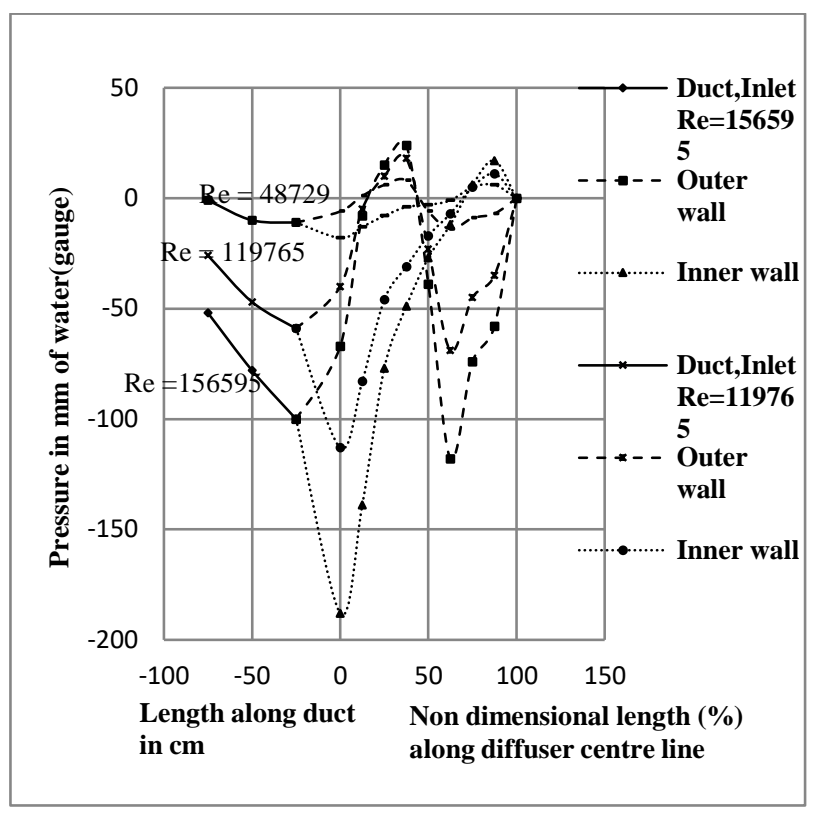

Figure 3. Pressure distribution curve for AR-2.5, $120^{\circ}$

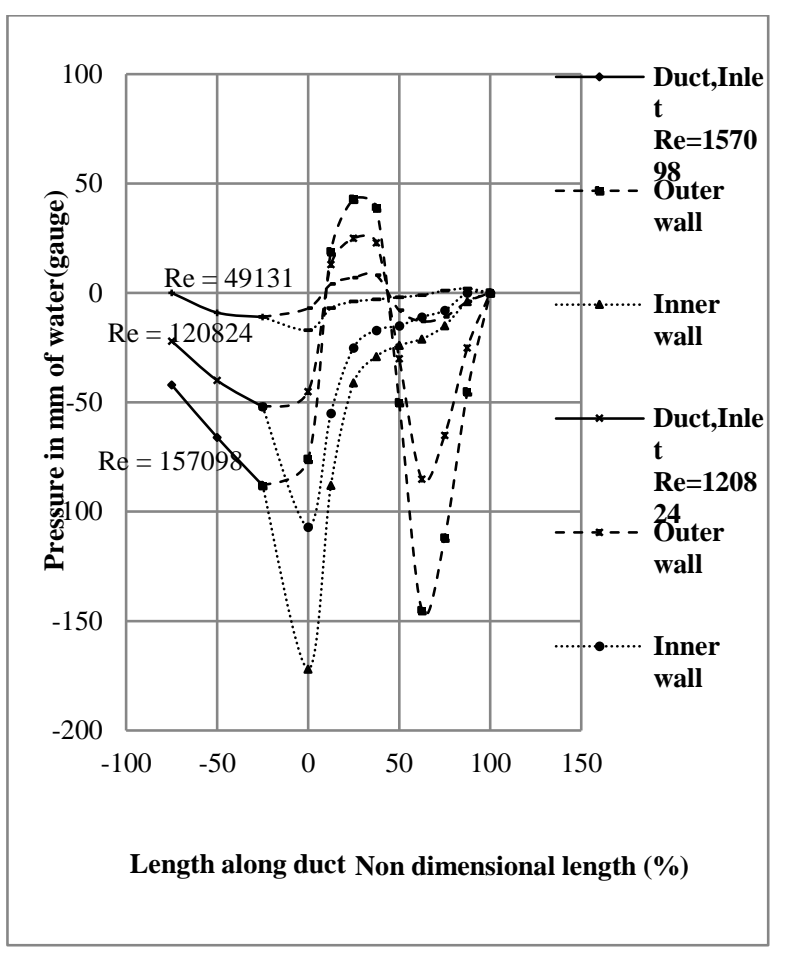

Figure 4. Pressure distribution curve for AR-3.5, $120^{\circ}$

As seen from fig. 4 for $\mathrm{AR}=3.5, \Delta \beta=120^{\circ}$ pressure on outer wall continuously increases till $25 \%$ of non dimensional length. For lower Re it increases upto $37.5 \%$ of non-dimensional length. After that pressure continuously decreases till $62.5 \%$ of non dimensional length and reaches 
below atmospheric and then again increases up to atmospheric. On inner wall it continuously increases up to atmospheric pressure. Up to $25 \%$ non dimensional length pressure recovery is maximum and after that it is very small. The nature of the curve is same for all 10 Reynolds number.

For $\mathrm{AR}=4.5$ and $\Delta \beta=120^{\circ}$, pressure distribution curve is shown in Fig. 5. Pressure on outer wall increases till $25 \%$ of non dimensional length. Then decrease and reaches below atmospheric till $62.5 \%$ of non dimensional length. After that it again increases till atmospheric pressure. On inner wall pressure recovery is maximum till $12.5 \%$ of non dimensional length and then very small change in pressure takes place till atmospheric condition is achieved. Nature of curve is similar for all ten Reynolds number.

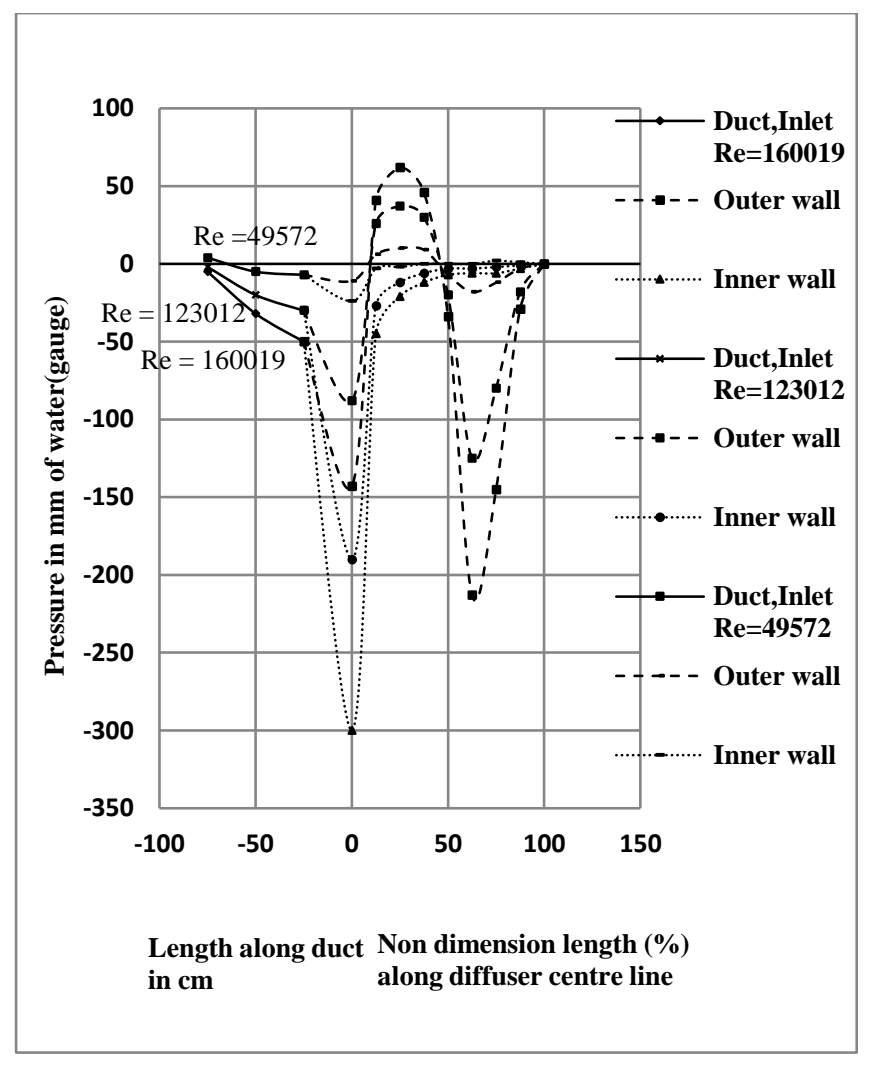

Figure 5. Pressure distribution curve for AR-4.5, $120^{\circ}$
For $\mathrm{AR}=5.5$ and $\Delta \beta=120^{\circ}$, pressure variation is shown in Fig. 6. Pressure on outer wall first increases and reaches above atmospheric till $37.5 \%$ of non dimensional length and then decreases below atmospheric condition till $62.5 \%$ of non dimensional length. Lowest pressure was observed at $62.5 \%$ of non dimensional length, after this point pressure increases to reach the atmospheric condition. On inner wall pressure continuously increases to reach atmospheric condition. Till $25 \%$ of non dimensional length pressure recovery is maximum and then very small change till atmospheric condition is achieved. Nature of curve is similar for all ten Reynolds number.

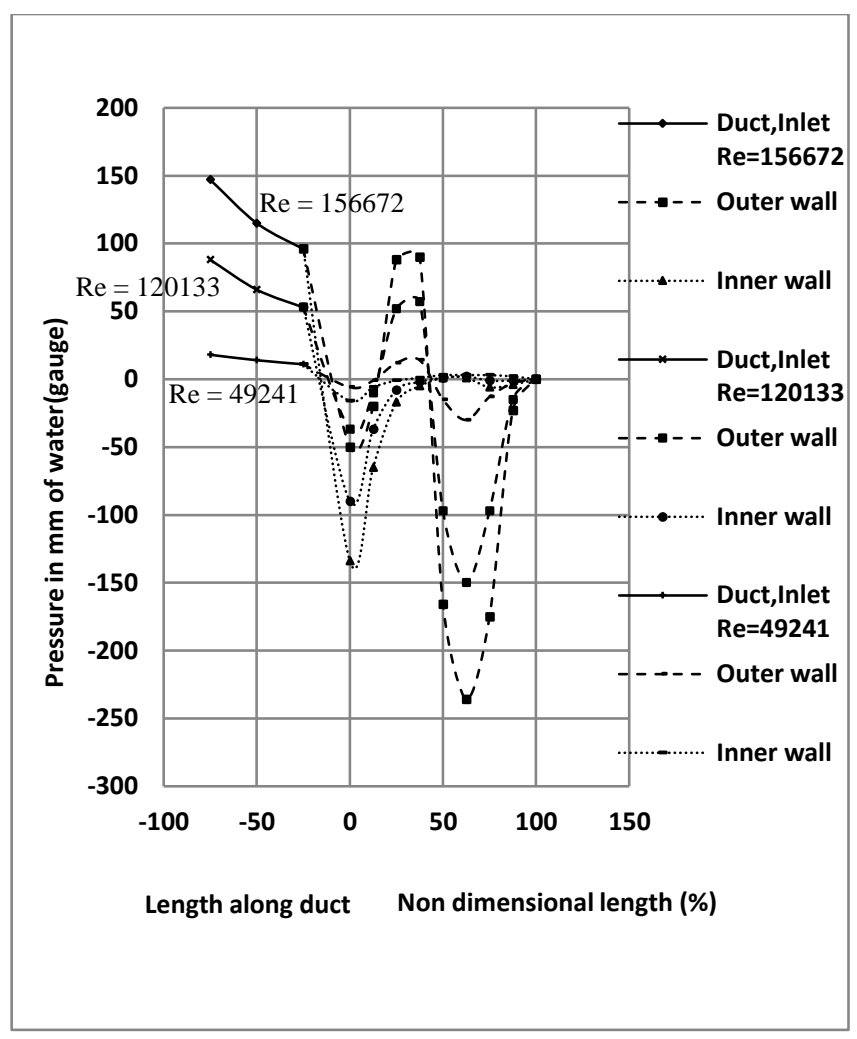

Figure 6. Pressure distribution curve for AR-5.5, $120^{\circ}$

For $\mathrm{AR}=6.5$ and $\Delta \beta=120^{\circ}$ pressure variation is shown in Fig. 7. The variation on outer and inner wall is same as described for $\mathrm{AR}=4.5$ and $\Delta \beta=120^{\circ}$ 
In all the five diffusers pressure on outer wall is more than the inner wall in first curvature and in second curvature pressure is higher on inner wall than outer wall. This is because direction of centrifugal force is towards outer wall in first curvature and towards inner wall in second curvature.

Effects of Reynolds number $(\mathrm{Re})$ on pressure recovery coefficient $\left(\mathbf{C}_{\mathbf{p}}\right)$ : There is not much change in value of $C_{p}$ within the range of test Reynolds number as evident from fig. 8. Also effect of area ratio can be seen. Value of $C_{p}$ first increases with increase in area ratio, reaches an optimum value and then decreases. No advantage is gained if $\mathrm{AR}$ is increased beyond this value.

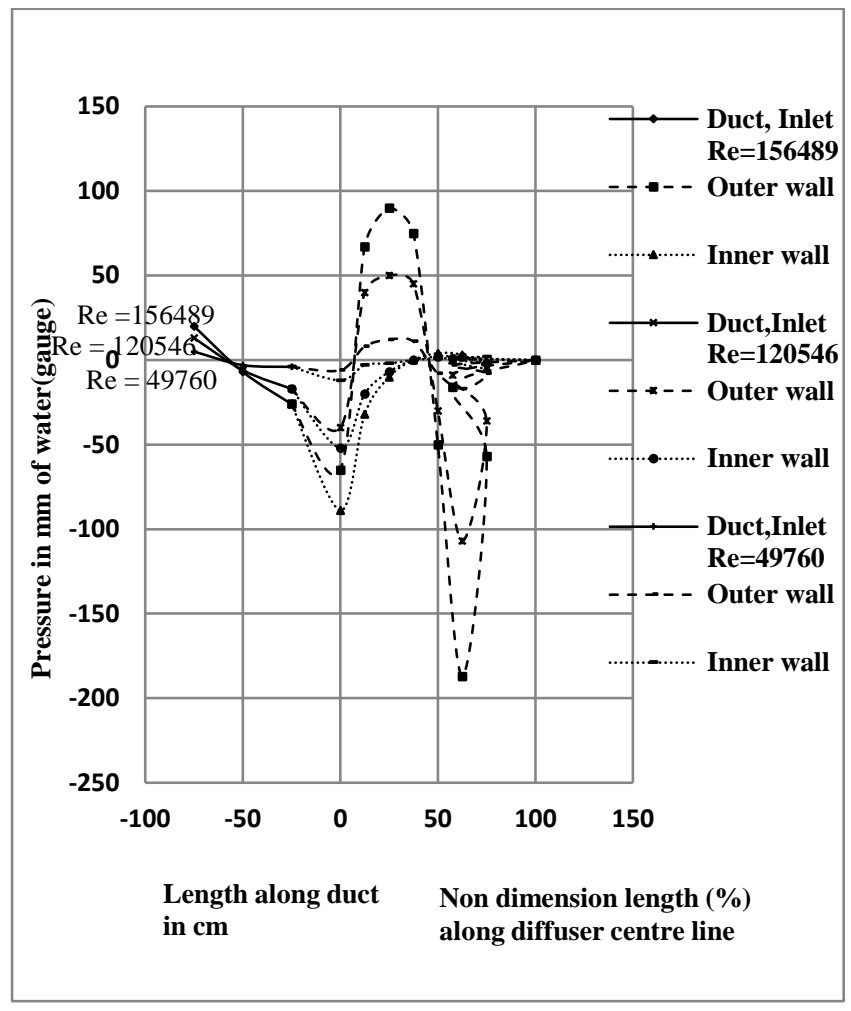

Figure 7. Pressure distribution curve for AR-6.5, $120^{\circ}$

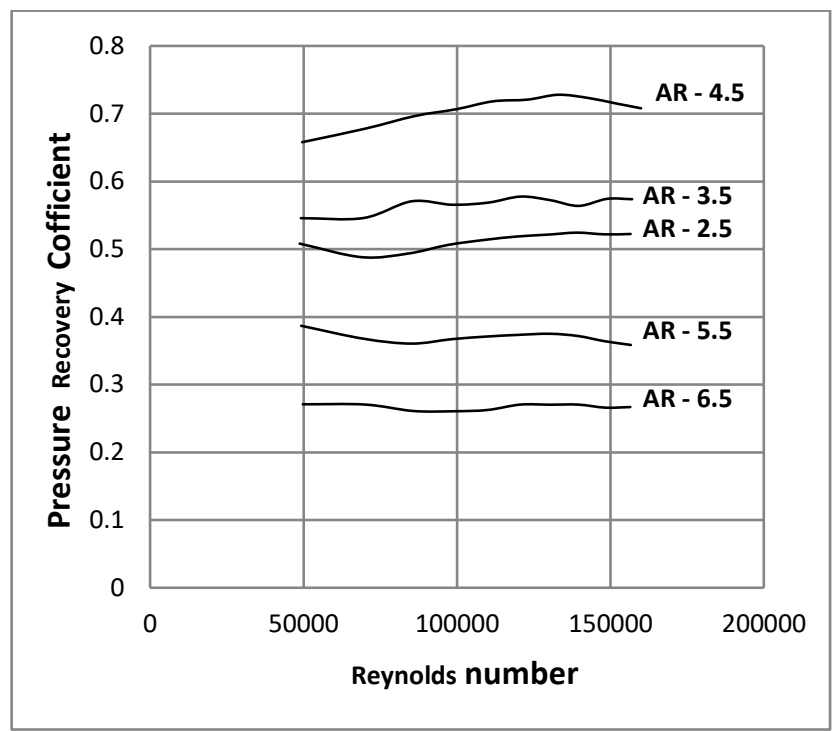

Figure 8. Pressure Recovery Coefficient Vs Reynolds Number for $120^{\circ}$ diffuser

Effects of Reynolds number $(\mathrm{Re})$ on pressure recovery efficiency $(\eta)$ :

From Fig. 9 it is evident that variation of $\eta$ with $\operatorname{Re}$ is similar as that of $C_{p}$ with Re. The value of $\eta$ are higher than corresponding $C_{p}$ values by a fixed constant for a particular area ratio. The value of constant changes when the area ratio is changed.

From Figs. 8 and 9 it is clear that variation in $C_{p}$ and $\eta$ is not much within the range of Reynolds number tested for a particular diffuser. This is because for the higher Reynolds number flows the flow is completely turbulent at the diffuser inlet and our range of test Reynolds number is much higher. There is a significant low Reynolds number effect on performance reported by earlier investigators. This drop in performance with decreasing Reynolds number below a critical value is attributed to a laminar separation immediately downstream of the diffuser throat. 


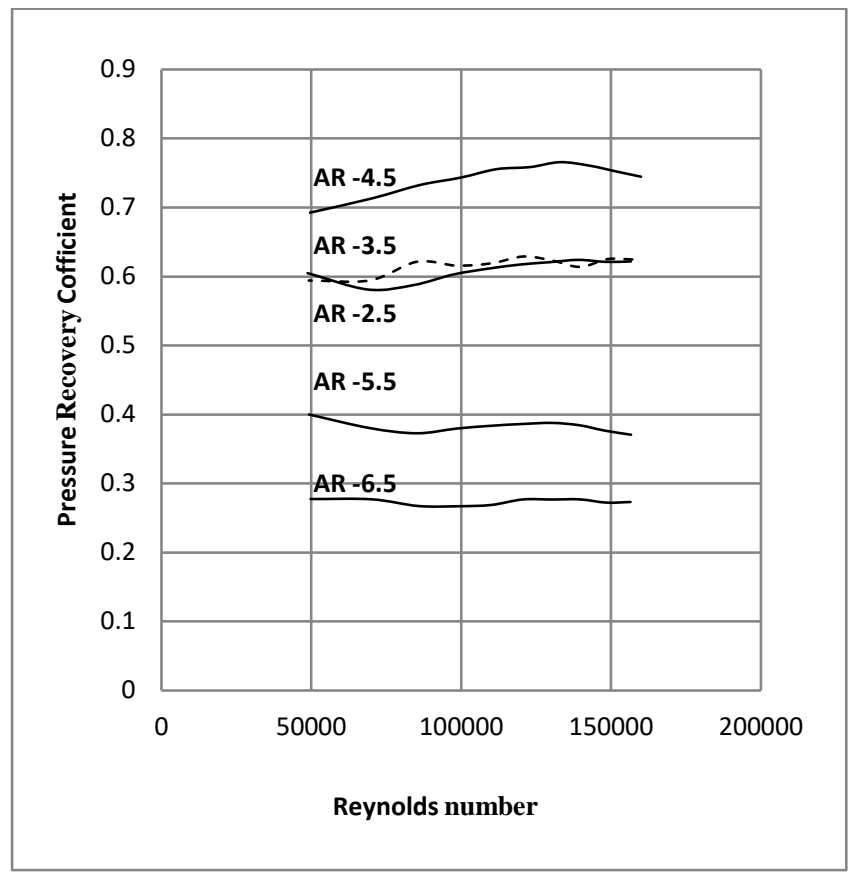

Figure 9. Pressure Recovery Efficiency Vs Reynolds Number for $120^{\circ}$ diffuser

From the result, it is clear that $C_{p}$ and $\eta$ increases with increase in area ratio but after a certain limit it decreases. $C_{p}$ and $\eta$ increases up to $A R=4.5$, after that it decreases. This is because of separation of flow near the diffuser wall. This is undesirable as losses will be more which will affect the $\eta$ of diffuser. The losses associated with the flow through the diffuser can be divided generally into two parts-losses due to friction and expansion losses. Except for small expansion $(2 \varphi$ less than $8^{\circ}$ ) the frictional losses are negligible as compared to expansion losses. As the area ratio or expansion angle of diffuser increases, expansion losses also increases rapidly as they are associated with the flow separation that occurs at the boundaries. As the area ratio or expansion angle is further increased, the fluid near the boundaries does not have sufficient kinetic energy to move against the adverse pressure gradient and back flow occurs. This results in high shearing stress in the fluid and losses associated become higher. In curved diffuser there is an additional loss due to bending of axis.

\section{Conclusions}

1. The pressure on the outer wall is more than that on the inner wall in the first curvature and less in the second curvature for all the diffusers tested, because the direction of centrifugal force is towards outer wall in the first curvature and towards inner wall in second curvature.

2. Variation in actual pressure recovery coefficient $(\mathrm{Cp})$ and pressure recovery efficiency $(\eta)$ is not very much within the range of Reynolds number tested for a particular diffuser because the flow is completely turbulent within the range or Reynolds number tested.

3. As area ratio increases value of $C p$ and $\eta$ also increases. As further increase in area ratio decreases $\mathrm{Cp}$ and $\eta$ because of expansion losses.

4. The optimum area ratio for 120 diffusers is 4.5 .

\section{Conflict of interest}

The author declares no conflict of interest.

\section{References}

[1] Galle K.R. and Binder, R.C., "Twodimensional flow Through a Diffuser with an Exit Length “, Journal of applied Mechanics, Trans. ASME, Vol. 75, pp. 390-392, 1953.

[2] Kline, S.J, "On the Nature of Stall”, Journal of Basic Engineering, Trans. ASME, Vol. 81, pp.305-320., September 1959.

[3] Kline, S.J., Abott, D.E., and Fox, R.W., "Optimum Design of Straight - Walled 
Diffusers", Journal of Basic Engineering, Trans. ASME, Vol. 81, pp. 321 - 331, September 1959.

[4] Waitman, B. A., Reneau, L.R. and Kline, S.J., "Effects of Inlet conditions on Performance of Two-Dimensional Subsonic Diffusers", Journal of Basic Engineering, Trans. ASME, Vol. 83, pp.349-360, September 1961.

[5] Carlson, J.J., Johnston, J.P., and Sagi, C.J., "Effects of Wall shape on Flow Regimes and performance in Straight, Two Dimensional Diffusers", Journal of Basic Engineering, Trans. ASME,Vol. 89, ,pp. 151 - 160, March 1967.

[6] Senoo, Y. and Nishi, M., "prediction of Flow Separation in a Diffuser by a Boundary Layer Calculation", Journal of Fluidsc Engineering, Trans. ASME, Vol. 99, pp.379-389, June 1977.

[7] Hoffmann, J. A., September, "Effects of FreeStream Turbulence on Diffuser performance", Journal of Fluids Engineering, Trans. ASME, Vol. 103, pp. 385-390, 1981.

[8] Thakur, S.N.,Vyas, B.D. and powle, U.S., December $15-17$, "Determination of Optimum Geometries for Straight Square Diffusers", proceedings of $16^{\text {th }}$ National Conference on Fluid Mechanics and Fluid power, Kanpur, India, pp. 23-27, 1988.

[9] Ward-Smith, A.J., Lane, D.J., Reynolds, A.J., Sahin, b. and Shawe, D.J., "Flow Regimes in Wide Angle Screened Diffusers", International Journal of Mechanical Sciences., Vol 33, No.1, pp. 41-54, 1991.

[10] Fox, R.W.and Kline, S.J., September, "Flow Regimes in curved Subsonic diffusers", Journal of basic Engineering, Trans, ASME, Vol. 84, pp.303-316, 1962.

[11] Sagi, C.J. and Johnston, J.P., "The Design and Performance of Two-Dimensional, Curved Diffusers", Journal of Basic Engineering, Trans. ASME, Vol. 89, pp. 715-731, December, 1967. [12] Thakur, S.N., Powle, U.S.and Vyas,B.D.," Flow Regimes in straight and Curved Square Diffusers", FLUCOME'91, ASME pp. 663-668, 1991.

[13] Mazumdar, B., Singh, S.N. and Agrawal, D.P., "Mean Flow Measurement in a Large Area ratio Curved Diffuser", Proceedings of $19^{\text {th }}$ National Conference on Fluid mechanics and Fluid power, 3-5, IIT Bombay, pp. HI-1 to HI-6, December 1992.

[14] Mazumdar, B. Mohan, R., Singh, S.N. and Agrawal, D.P.," Experimental Study of Flow in a High Aspect Ratio 90 Curved Diffuser", Journal of Fluids Engineering, Trans ASME, Vol. 120, pp.83-89, March, 1998.

[15] Weng, P. F and Guo, R. W., 'New Method of Swirl Control in a Diffusing Sduct', AIAA Journal, Vol. 30, No. 7, pp. 1918 - 1919, 1992.

[16] Tabhane, V., " Investigation on Subsonic S - Shaped Diffusers with Constant Width and Square Cross Section at Inlet', M.Tech. Project Report, Dept. of Mech. Engg., I.I.T., Bombay, 1997.

[17] Varshney, R., 'Investigation on S-Shaped Diffusers', M. Tech. Experimental Engineering Project Report, Dept. of Mech. Engg., I.I.T. Bombay, 2000. 